\title{
Measurement of the third order nonlinear susceptibility of paratellurite single crystal using multiplex CARS
}

Cite as: AIP Advances 9, 105301 (2019); https://doi.org/10.1063/1.5113478

Submitted: 04 June 2019. Accepted: 27 September 2019 . Published Online: 03 October 2019

Zakaniaina Rajaofara, Philippe Leproux, Erwan Capitaine (D), Hideaki Kano, Tomokatsu Hayakawa (D), Philippe Thomas (D), Jean-René Duclère, and Vincent Couderc

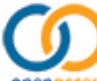

\section{AVS Quantum Science}




\title{
Measurement of the third order nonlinear susceptibility of paratellurite single crystal using multiplex CARS
}

Cite as: AIP Advances 9, 105301 (2019); doi: 10.1063/1.5113478

Submitted: 4 June 2019 Accepted: 27 September 2019 •

Published Online: 3 October 2019

Zakaniaina Rajaofara, ${ }^{1,2}$ Philippe Leproux, ${ }^{2}$ Erwan Capitaine, ${ }^{2}$ (D) Hideaki Kano, ${ }^{3}$ Tomokatsu Hayakawa, Philippe Thomas,' (D) Jean-René Duclère, ${ }^{,}$and Vincent Couderc ${ }^{2, a)}$

\begin{abstract}
AFFILIATIONS
'Institut de Recherche sur les Céramiques, UMR 7315 CNRS-Université de Limoges, Centre Européen de la Céramique, 12, rue Atlantis, 87068 Limoges Cedex, France

2Institut XLIM, UMR 7252 CNRS - Université de Limoges, 123, Avenue Albert Thomas, 87060 Limoges Cedex, France

${ }^{3}$ Department of Applied Physics, Graduate School of Pure and Applied Sciences, University of Tsukuba, 1-1-1 Tennodai, Tsukuba, Ibaraki, 305-8573, Japan

${ }^{4}$ Field of Advanced Ceramics, Department of Life Science and Applied Chemistry, Nagoya Institute of Technology, Gokiso, Showa, Nagoya 466-8555, Japan
\end{abstract}

a) Corresponding author: vincent.couderc@xlim.fr

\begin{abstract}
We report the extraction of the real part of the third order nonlinear susceptibility for a c-cut paratellurite $\left(\mathrm{TeO}_{2}-\alpha\right)$ single crystal using the nonresonant contribution of the multiplex coherent anti-Stokes Raman scattering (M-CARS) signal. Using fused silica and SF57 as nonlinear reference materials, we derive the absolute value of the real part of the electronic third order susceptibility and we evidence the in-plane modulation of the nonlinear refractive index. These results are in total agreement with those recently obtained by the $\mathrm{z}$-scan method.

(C) 2019 Author(s). All article content, except where otherwise noted, is licensed under a Creative Commons Attribution (CC BY) license (http://creativecommons.org/licenses/by/4.0/). https://doi.org/10.1063/1.5113478
\end{abstract}

\section{INTRODUCTION}

The coherent Raman anti-Stokes scattering (CARS) technique has been commonly used for microscopy applications thanks to its wide ability to probe the vibrational resonances of biological samples. The first measurement of CARS radiation has been obtained in 1965 by P. D. Maker and R. W. Terhune ${ }^{1}$ after the theoretical prediction published by J. A. Armstrong et al. in $1962 .{ }^{2}$ In its simplest configuration, CARS system is used to probe a single vibrational mode which is stimulated by the beating of two monochromatic synchronized waves called pump $\left(\omega_{p}\right)$ and Stokes $\left(\omega_{s}\right)$ waves. When the power density exciting the sample is sufficiently strong and the frequency difference between pump and Stokes waves matches that of a vibrational mode, a coherent response from the matter is observed. The nonlinear mechanism is driven by a four-wave mixing process delivering an anti-Stokes wave $\left(\omega_{a s}\right)$ which satisfies the energy conservation law: $\omega_{p}+\omega_{p}-\omega_{s}=\omega_{a s}$. More recently dual broadband CARS has been demonstrated allowing simultaneous identification of several vibrational modes. ${ }^{3}$ Later, Zumbusch et $a$ l $^{4}$ implemented CARS in a microscope for vibrational imaging of chemical and biological samples. Then multiplex CARS (M-CARS) has been introduced by using supercontinuum generated in microstructured optical fibers. CARS spectra covering more than $3500 \mathrm{~cm}^{-1}$ have been obtained by this method on a large diversity of samples. ${ }^{5,6}$ Systematically, M-CARS vibrational signatures are accompanied by an additional nonlinear signal called nonresonant background (NRB), which is provided by the electronic response of the matter. The NRB is coherent with the vibrational signal and thus strongly modulates the total CARS intensity which is given by the Eq. 1: 


$$
I_{C A R S}(\omega) \propto\left|\chi_{N R}^{(3)}(\omega)\right|^{2}+\left|\chi_{R}^{(3)}(\omega)\right|^{2}+2 \mathfrak{R} e\left[\chi_{N R}^{(3)}(\omega)\right] \mathfrak{R} e\left[\chi_{R}^{(3)}(\omega)\right]
$$

where $\chi_{N R}^{(3)}$ and $\chi_{R}^{(3)}$ are the nonresonant and the resonant complex third order nonlinear susceptibilities respectively.

The $\chi_{N R}^{(3)}$ term introduces a broadband response clearly visible on the entire M-CARS spectra. In most cases, vibrational signature is extracted from the spectra by using phase retrieval algorithms. ${ }^{8,9}$ As well, experimental approaches have been developed to reduce or suppress the NRB in M-CARS configuration. ${ }^{10,11}$ On another side, in the case where no vibrational contribution arises and where the multi photon absorption is neglected, the CARS intensity is only feeded by the nonlinear electronic contribution with a direct relation with the square of the real part of the third order susceptibility (or Kerr susceptibility) as shown in Eq. 2. Then measurement of Kerr response becomes possible with a M-CARS system.

$$
I_{C A R S}(\omega) \propto\left[\Re e\left(\chi_{N R}^{(3)}(\omega)\right)\right]^{2}
$$

In this paper we present the extraction of the real part of the electronic response $\mathfrak{R} e\left[\chi_{N R}^{(3)}(\omega)\right]$ of a c-cut $\mathrm{TeO}_{2}-\alpha$ single crystal by considering the NRB of M-CARS spectra. The absolute value of the nonlinear response is obtained by comparison with fused silica $\mathrm{SiO}_{2}$ and Schott N-SF57 glasses used as standard nonlinear media with known nonlinear parameters. We show that the pure nonlinear electronic contribution can be obtained by a broadband spectral analysis excluding the vibrational region, where dispersion of $\chi_{R}^{(3)}$ is observed. ${ }^{12}$ The results are then compared with the recent data obtained by Duclère et al. from z-scan experiments. ${ }^{13}$ Finally, we evaluate the in-plane modulation of the nonlinear refractive index of $\mathrm{TeO}_{2}-\alpha$.

\section{EXPERIMENTAL SETUP}

The multiplex CARS setup is presented in Fig. 1. The source is a Sirius Spark Laser which delivers 60 ps pulses at $1064 \mathrm{~nm}$ with $150 \mathrm{kHz}$ repetition rate. The laser beam is split into two

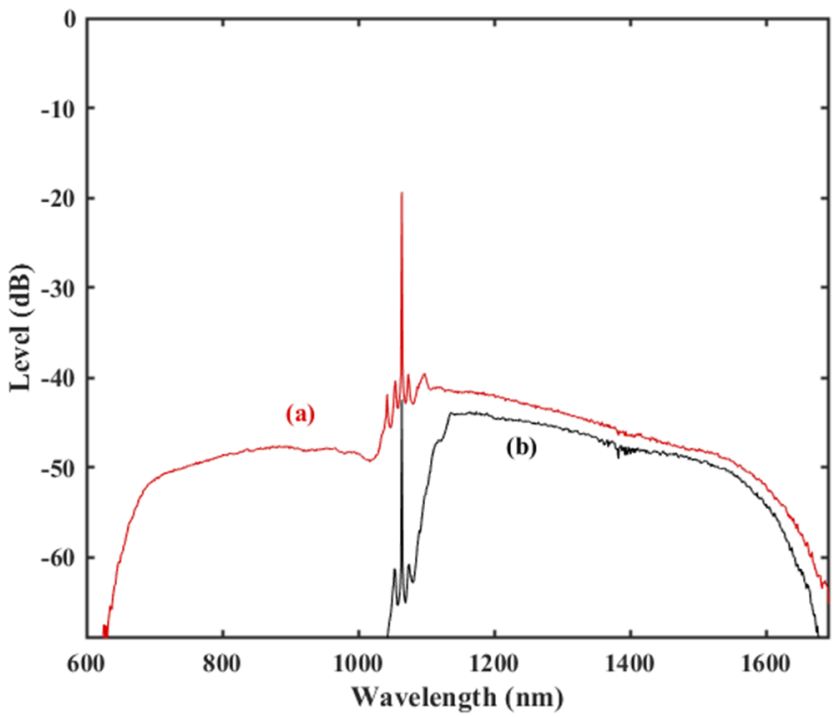

FIG. 2. Broadband spectrum of the Stokes beam (a) at the output of the PCF and (b) after the excitation objective (Olympus UplanSAPo x60).

parts by using a half-wave plate and a Glan-Taylor polarizer. The first part is injected in a photonic crystal fiber (PCF) to generate a supercontinuum spectrum between $600 \mathrm{~nm}$ and $1700 \mathrm{~nm}$ (see Fig. 2).

A long pass filter (Thorlabs, FEL1050) selects its infrared range between $1050 \mathrm{~nm}$ and $1700 \mathrm{~nm}$ to be used as the Stokes wave. The second part is used as pump wave which is sent in a delay line to compensate the propagation in the nonlinear microstructured optical fiber undergone by the broadband Stokes wave. The two incident beams are then recombined by means of a dichroic mirror (Semrock, NFD01-1064-25x36). Both Stokes and pump are randomly and linearly polarized respectively and are focused at the sample by a microscope objective (Obj 1: Olympus, UPlanSApo 60x, N.A. $=1.2$, water immersion). This water immersion objective is used to maximize the numerical aperture and to ensure a high spatial

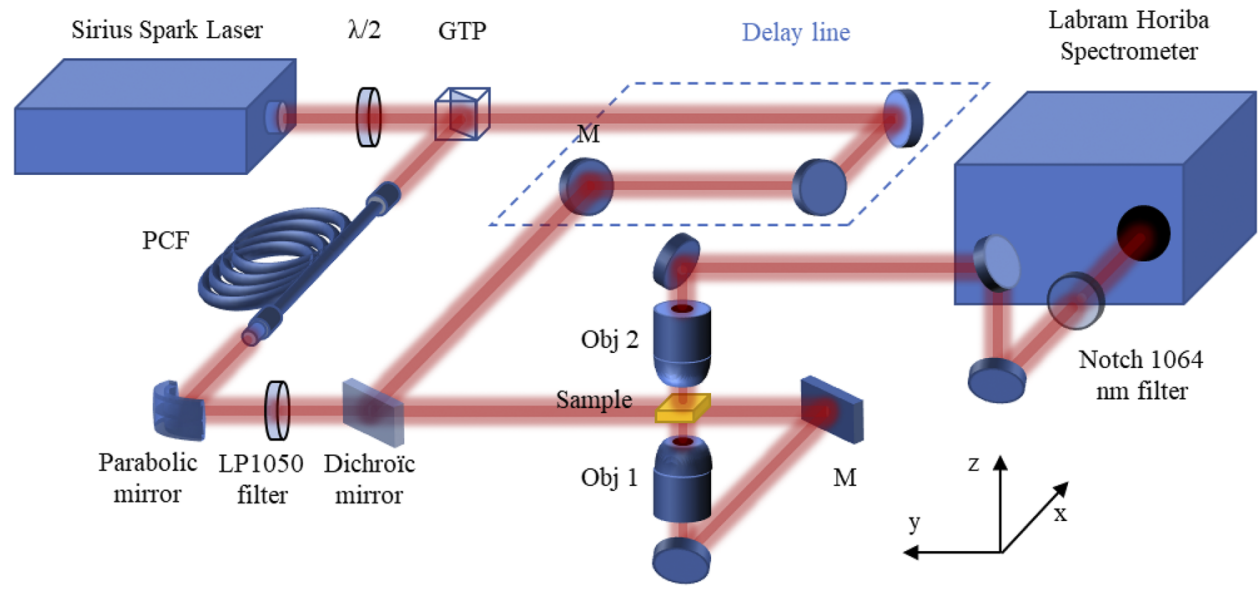

FIG. 1. CARS experimental setup. $\lambda / 2$ half-wave plate; GTP, Glan-Taylor polarizer; PCF, photonic crystal fiber. 


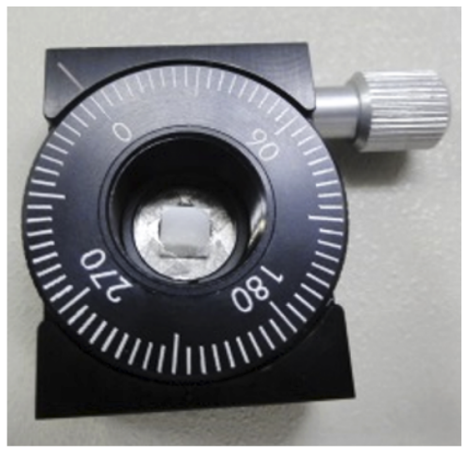

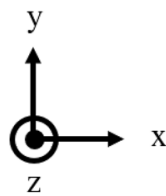
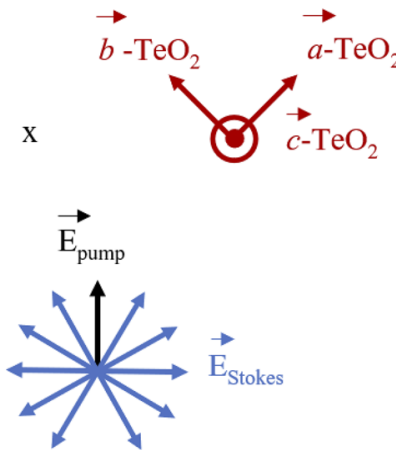

FIG. 3. $\mathrm{TeO}_{2}-\alpha$ crystal sample orientation with respect to its crystallographic axes $\vec{a}-\mathrm{TeO}_{2}, \vec{b}-\mathrm{TeO}_{2}$ and $\vec{c}-\mathrm{TeO}_{2}$ (red arrows). The Stokes is randomly polarized (blue arrows) while the pump polarization is along y axis (black arrow). resolution. Because of the nature of the crystal, no detrimental interaction between the sample and the immersion fluid (Zeiss, Immersol W 2010) is observed and, the focus plane being in the crystal, no parasitic CARS signal from the immersion fluid is generated. The measured power of the pump behind the excitation objective is 16.1 $\mathrm{mW} \pm 0.1 \mathrm{~mW}$ and that of Stokes is $5.8 \mathrm{~mW} \pm 0.1 \mathrm{~mW}$. The antiStokes beam is then collected by a second microscope objective (Obj 2: Nikon, S Plan Fluor ELWD 60x, N.A.=0.7) before being sent into the spectrum analyzer (CCD Horiba, Synapse) with a maximum resolution of $1 \mathrm{~cm}^{-1}$.

The sample is a c-cut paratellurite single crystal with a helical structure and four-fold symmetry properties ${ }^{14}$ set on a manual rotation stage. As a consequence, data will be collected over a $\left[0^{\circ}-360^{\circ}\right]$ range using a rotation step of $10^{\circ}$. Then, they will be averaged and rescaled on a $\left[0^{\circ}-90^{\circ}\right]$ range $\left(90^{\circ}\right.$ being strictly equivalent to $0^{\circ}$ by symmetry). Its two crystallographic axes labeled $\vec{a}-\mathrm{TeO}_{2}$ and $\vec{b}-\mathrm{TeO}_{2}$ are referenced in the rotating mount at $90^{\circ}$ and $0^{\circ}$ respectively (see Fig. 3 ). The initial polarization direction of the pump beam is oriented at $45^{\circ}$ along the bisector of $\vec{a}-\mathrm{TeO}_{2}$ and $\vec{b}-\mathrm{TeO}_{2}$

\section{RESULTS AND DISCUSSION}

\section{A. Extraction of $\Re e\left[\chi_{N R}^{(3)}\right]$}

The broadband traces of $\mathrm{TeO}_{2}-\alpha$ CARS spectra for different rotation stage angles are given in Fig. 4 where the anti-Stokes intensity is plotted versus the wavenumber. In this figure, a single vibrational signature of $\mathrm{TeO}_{2}-\alpha$ is recorded at $644 \mathrm{~cm}^{-1}$ and is assigned to the stretching modes of the equatorial $\mathrm{Te}-\mathrm{O}$ bonds in $\mathrm{TeO}_{4}$ trigonal bipyramid units. ${ }^{15}$ In all the remaining frequency range, only the NRB is still visible. It is also important to underline that in our case, the vibrational signature of the $\mathrm{TeO}_{2}-\alpha$ crystal is significantly high compared to the NRB signal. In these conditions, and to avoid any parasitic effect of the vibrational signature, the NRB signature used to extract the $\mathfrak{R} e\left[\chi_{N R}^{(3)}\right]$ is exploited only for wavenumbers beyond $1000 \mathrm{~cm}^{-1}$. The output signal level is then quadratically dependent on the pump inensity and linearly dependent on the Stokes intensity at each wavelength according to Eq. 3.

$$
I_{C A R S}(\omega) \propto\left[\Re e\left(\chi_{N R}^{(3)}\right)\right]^{2} I_{p}(\omega)^{2} I_{s}(\omega)
$$

The raw anti-Stokes wave intensity obtained at the sample output is, thus, divided by the Stokes wave spectral profile (see Fig. 4) to get rid of the initial supercontinuum variation. The normalized experimental data show, at first glance, that the antiStokes intensity seems to be roughly constant between $1000 \mathrm{~cm}^{-1}$ and $3000 \mathrm{~cm}^{-1}$. In fact, a carefull observation clearly reveals that there is a moderate evolution of the NRB with respect to the crystal in-plane orientation (See further discussion in Sec. III B).

In order to extract a quantitative mean value of the nonlinear susceptibility of the paratellurite crystal, we first averaged all the curves obtained for different rotation stage angles and compared them with those obtained for fused silica and SF57 samples. The comparison is presented on Fig. 5 in log scale.

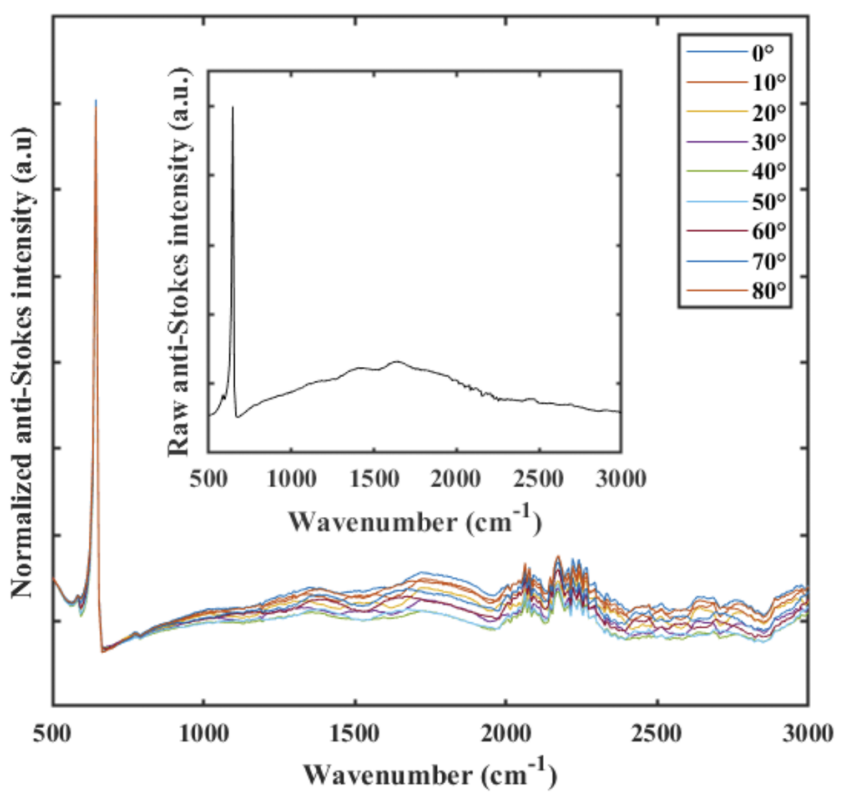

FIG. 4. CARS spectra of $\mathrm{TeO}_{2}-\alpha$ normalized by the Stokes broadband spectrum for different rotation stage angles. Inset: Example of raw CARS spectrum of $\mathrm{TeO}_{2}$ $-\alpha$ for a given position of the rotation stage. 

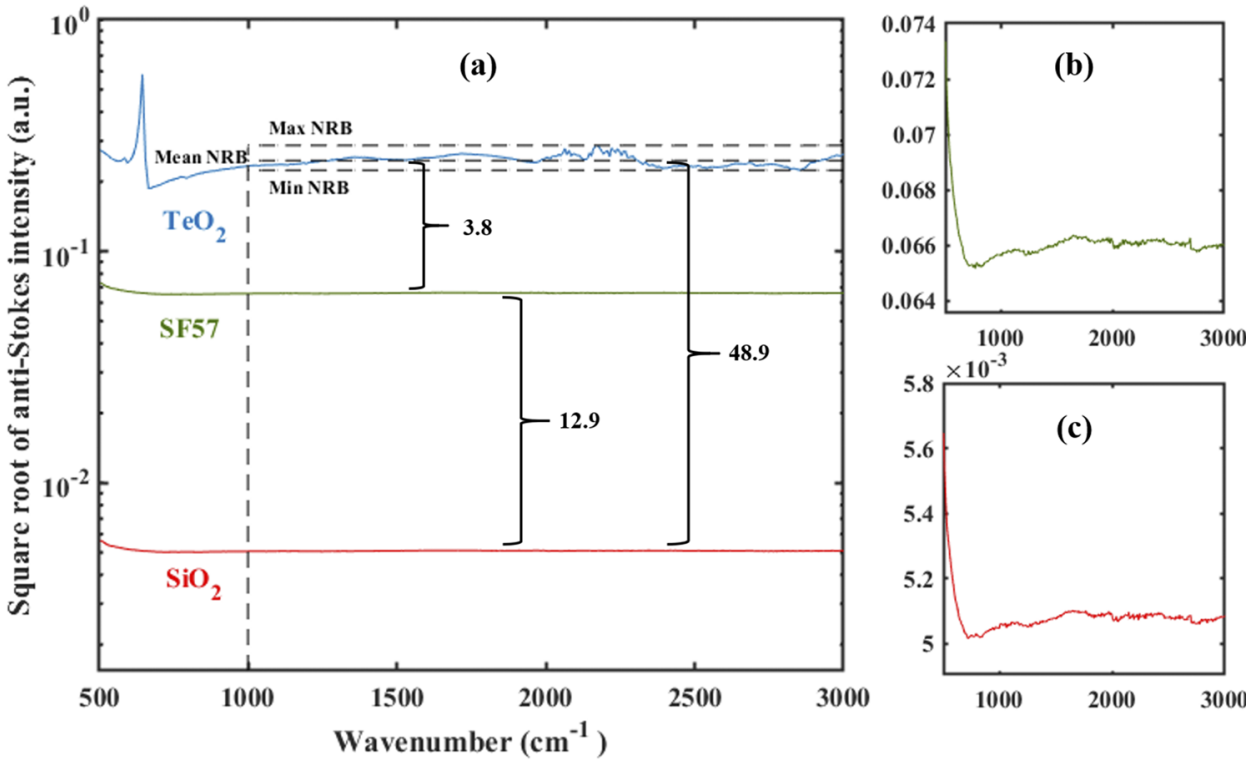

FIG. 5. Comparison of the square roo of CARS NRB level of fused silica (red curve), SF57 (green curve) and $\mathrm{TeO}_{2}-\alpha$ (blue curve) in log scale (a). Zoomed-in CARS spectra of SF57 (b) and $\mathrm{SiO}_{2}$ (c). Black dashed lines delimitate the spectral range of the NRB between $1000 \mathrm{~cm}^{-1}$ and $3000 \mathrm{~cm}^{-1}$ that was used to extract the mean $n_{2}$ value.

TABLE I. Comparison of the ratio $\frac{\mathfrak{\Re e}\left[\chi^{(3)}\left(\mathrm{TeO}_{2}\right)\right]}{\mathfrak{\Re e}\left[\chi^{(3)}\left(\mathrm{SiO}_{2}\right)\right]}$ for different methods (Z-scan $\left.{ }^{13} / \mathrm{CARS} / \mathrm{THG}^{22}\right)$.

\begin{tabular}{lccc}
\hline \hline & Z-scan & CARS (this work) & THG \\
\hline$\lambda_{\text {pump }}(\mathrm{nm})$ & 800 & 1064 & 1900 \\
$\Re e\left[\chi^{(3)}\left(\mathrm{TeO}_{2}\right)\right]$ & 49.1 & 48.9 & 50.4 \\
$\Re \mathfrak{R e}\left[\chi^{(3)}\left(\mathrm{SiO}_{2}\right)\right]$ & $\left(\mathrm{TeO}_{2}-\alpha\right.$ crystal $)$ & $\left(\mathrm{TeO}_{2}-\alpha\right.$ crystal $)$ & (pure $\mathrm{TeO}_{2}$ glass) \\
&
\end{tabular}

As the nonlinear refractive index $n_{2}$ is proportional to $\Re e\left[\chi_{N R}^{(3)}\right]$ according to Eq. $4^{16}$

$$
n_{2}=\frac{3 \Re e\left[\chi_{N R}^{(3)}\right]}{4 n_{0}^{2} \varepsilon_{0} c}
$$

where $n_{0}$ is the linear refractive index, $c$ is the light velocity and $\varepsilon_{0}$ the vacuum permittivity, we deduced the value of $n_{2}$ of $\mathrm{TeO}_{2}-\alpha$. All our results and comparisons to the relevant literature values are summarized in Table I and Table II.

TABLE II. Values of nonlinear refractive index $n_{2}$ of $\mathrm{TeO}_{2}-\alpha$, fused silica and SF57.

\begin{tabular}{lcc}
\hline \hline Sample & $\lambda_{\text {pump }}(n m)$ & $n_{2}\left(m^{2} . W^{-1}\right)$ \\
\hline $\mathrm{SiO}_{2}{ }^{17}$ & 1053 & $0.274 \pm 0,17 \times 10^{-19}$ \\
$\mathrm{SF}^{18-21}{ }^{18}$ & 1064 & $2.25 \times 10^{-19}$ \\
$\mathrm{TeO}_{2}-\alpha^{13}$ (by Z-scan) & 800 & $5.86 \times 10^{-19}$ \\
$\mathrm{TeO}_{2}-\alpha$ (this work) & 1064 & $5.72 \pm 0.73 \times 10^{-19}$ \\
$\mathrm{SF}$ (this work) & 1064 & $2.27 \pm 0.14 \times 10^{-19}$ \\
\hline \hline
\end{tabular}

In our configuration, the square root of the mean NRB level of $\mathrm{TeO}_{2}-\alpha$ is 48.9 and 3.8 times higher than those of fused silica and SF57 respectively. In these conditions, the mean $n_{2}$ value of $\mathrm{TeO}_{2}-\alpha$ is estimated to be $5.72 \pm 0.73 \times 10^{-19} \mathrm{~m}^{2} W^{-1}$ at $1064 \mathrm{~nm}$ assuming the $n_{2}$ value of silica close to $0.274 \times 10^{-19} \mathrm{~m}^{2} \mathrm{~W}^{-1}$ at $1053 \mathrm{~nm} .{ }^{17}$ Then we can compare this value to the one recently reported by Duclère et al. ${ }^{13}$ which is $5.86 \times 10^{-19} \mathrm{~m}^{2} \mathrm{~W}^{-1}$ (measurement realised at $800 \mathrm{~nm}$ by using $\mathrm{z}$-scan technique in femtosecond domain for the exact same $\mathrm{TeO}_{2}-\alpha$ crystal). Excellent agreement is observed between both values. Finally, by using the same approach, the extracted value of SF57 is then $2.27 \pm 0.14 \times 10^{-19} \mathrm{~m}^{2} \mathrm{~W}^{-1}$, thus once again very close to the value of $2.25 \times 10^{-19} \mathrm{~m}^{2} W^{-1}$ resulting from the average of four different $n_{2}$ values reported in the literature. ${ }^{18-21}$

Hence, though the pulse duration used in our CARS experiment is much longer (60 ps) than the one used by Duclère et al. (90 fs), ${ }^{13}$ our results prove that a simple spectral filtering of the broadband anti-Stokes wave can remove the vibrational response of the material and successfully extract the real part of the pure electronic response of the third order nonlinear optical susceptibility. Thus, the experimental data are recorded for high wavenumbers (between 1000 and $3000 \mathrm{~cm}^{-1}$ ), far enough from the vibrational signature. 


\section{B. In-plane modulation of the nonlinear refractive index}

Third order nonlinear polarization in a given direction $i$ can be written as a function of the incident electric field $E_{j}, E_{k}$ and $E_{l}$ in the directions $j, k$ and $l$ according to Eq. 5 .

$$
P_{i}^{(3)}=\varepsilon_{0} \sum_{j k l} \chi_{i j k l}^{(3)} E_{j} E_{k} E_{l}
$$

$\chi_{i j k l}^{(3)}$ is an element of the $\chi_{N R}^{(3)}$ tensor which has some angular dependence.

For the same c-cut $\mathrm{TeO}_{2}-\alpha$ single crystal, the $\left(\vec{a}-\mathrm{TeO}_{2}, \vec{b}\right.$ $\mathrm{TeO}_{2}$ ) in-plane angular dependence of the nonlinearity was recently assessed by Duclère et al., ${ }^{13}$ via the z-scan technique. While rotating the crystal around the four-fold symmetry c-axis, the in-plane modulation of the $\chi^{(3)}$ was clearly evidenced as depicted in Fig. 6b through the amplitude variation of the normalized transmittance $\Delta T_{p-v}$ (converted here in values of $n_{2}$ for the comparison) and, in particular, the values of some elements of the $\chi^{(3)}$ tensor (namely $\mathfrak{R} e\left[\chi_{x x x x}^{(3)}\right]$ and $\left.\mathfrak{R} e\left[\chi_{x x y y}^{(3)}\right]\right)$ were derived. ${ }^{13}$

Hence, in addition to the relative values of nonlinear refractive index discussed in Sec. III A, it is also expected to access the same angular dependence within the $\left(\vec{a}-\mathrm{TeO}_{2}, \vec{b}-\mathrm{TeO}_{2}\right)$ plane, thanks to our CARS setup. We have seen in Eq. 2 that CARS intensity is proportional to the square of $\mathfrak{R}\left[\chi_{N R}^{(3)}\right]$, provided that it is recorded far enough (between $1000 \mathrm{~cm}^{-1}$ and $3000 \mathrm{~cm}^{-1}$ ) from any vibrational response of the medium. We then recorded in this range the mean evolution of the NRB intensity and plotted its square root for different rotation stage angles from $0^{\circ}$ to $90^{\circ}$. So Fig. 6 reveals, indeed, that CARS data present such modulation of the nonlinear refractive index $n_{2}$ with the rotation of the stage (obviously, such modulation is totally absent for both silica and SF57 reference glass samples). The modulation depth reaches $\sim 15.1 \%$, whereas the one deduced from $\mathrm{z}$-scan experiments was $\sim 11.5 \%$. Again, results from CARS experiments fall within the same range as those obtained from z-scan experiments. The differences could be related, of course, to the experimental error but also to the spectral dependence of the nonlinear refractive index as the $\mathrm{z}$-scan experiment was conducted at $800 \mathrm{~nm}$, whereas the current CARS measurement is runned with a pump at $1064 \mathrm{~nm}$.
Further analysis focuses on the angular positions of the maximum and minimum observed in Fig. 6. Based on the specific experimental configuration described in Fig. 1, where the pump laser is linearly polarized along the y direction, the latter forming a $45^{\circ}$ angle with the $\vec{b}-\mathrm{TeO}_{2}$ and $\vec{a}-\mathrm{TeO}_{2}$ lattice vectors, and as previously discussed in details by Duclère et al., ${ }^{13}$ respective positions of the maximum and minimum of the in-plane nonlinear response are theoretically expected along the $<110>$ and $<100>$ directions of the crystal lattice. Additionally, paratellurite material is known for being optically active. ${ }^{14,23}$ Indeed, the polarization direction of the laser beam can progressively dextro-rotate along the propagation across the thickness of the crystal, and emerge from the latter with a rotation angle $\rho$ (given in $\% / \mathrm{mm}$ ), according to a law modelised by Kolesnikov et al. ${ }^{23}$ and summarized here with the Eq. 6:

$$
\rho^{-1}=a+b \lambda^{2} \ln (\lambda)
$$

where $\lambda$ is the wavelength (in Angströms) and a and $b$ are two known parameters $\left(a=-0.00310\right.$ and $\left.b=4.17 \times 10^{-11}\right)$. Thus, for previous $\mathrm{z}$-scan experiments (Fig. $6 \mathrm{~b}$ ), the respective positions for the maximum and minimum intensities are shifted in respect to $0^{\circ}$ and $45^{\circ}$, because the beam waist crosses the whole sample thickness, and consequently the known optical activity of paratellurite ${ }^{14,23}$ must be taken into account. ${ }^{13}$ Considering the optical activity of such $\mathrm{TeO}_{2}$ $-\alpha$ crystal, with a thickness of $l=565 \mu m$ which induces a dextro rotation to $\vec{E}_{\text {pump }}$ by an angle $\rho$, one expects to detect a minimum of $\mathfrak{R} e\left[\chi_{N R}^{(3)}\right]$ for an angular position of $45^{\circ}+\rho l / 2$, instead of $45^{\circ} \cdot{ }^{13}$ The position of the minimum evidenced for the set of $z$-scan data theoretically falls at $\sim 58.9^{\circ}$ (at $800 \mathrm{~nm}$ ) and experimentally, the result of the fitting gives a minimum evidenced at $\sim 57.4^{\circ}$, which is in good agreement with the theory.

In our current CARS configuration, beside a very small beam waist (axial spot size $\leq 2 \mu \mathrm{m}$ ) by the use of x60 excitation objective, the focus plane of excitation beams is near the lower physical surface of the crystal. The thickness crossed by beam waist is, thus, very small against the crystal total thickness of $565 \mu \mathrm{m}$ and the optical activity can be neglected. Hence, the maximum and minimum CARS signals are respectively spotted at $\sim 0^{\circ}$ and $\sim 45^{\circ}$ (Fig. 6a), which is also in good agreement with the theory. Fig. 6 finally attests again the good matching between the two sets of data, and demonstrates the relevance of conducting such CARS experiments in the aim of accessing third-order nonlinear optical properties.
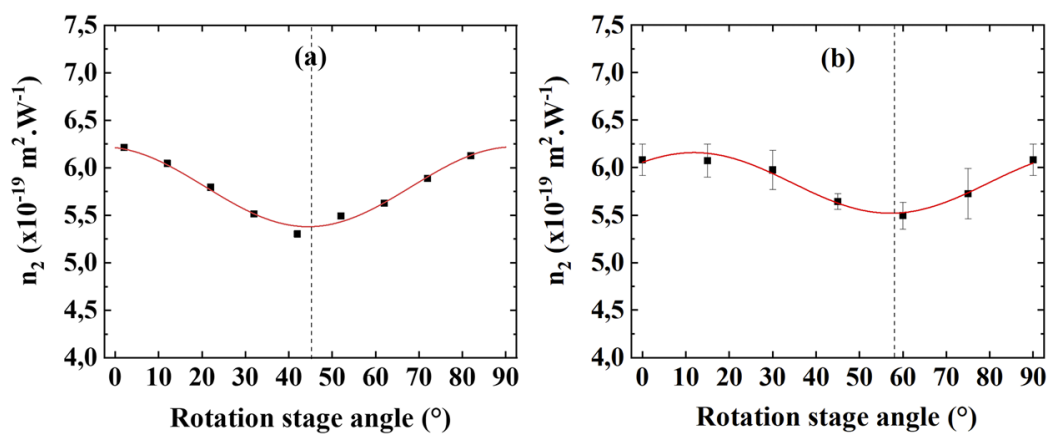

FIG. 6. In-plane angular dependence of the nonlinear refractive index $n_{2}$ in CARS experiments (a) in comparison with z-scan data (b). ${ }^{13}$ The angular position of the minimum value of $n_{2}$ is highlighted (black dashed line) in each case ( $45^{\circ}$ in CARS and $57.4^{\circ}$ in z-scan). Red line: sine fit of experimental data. 


\section{CONCLUSION}

In this study we demonstrated that the real part of the pure electronic response of a paratellurite single crystal $\mathrm{TeO}_{2}-\alpha$ can be obtained by using picosecond M-CARS experiment. By a broadband spectral analysis of the CARS intensity, the electronic contribution can be separated from the vibrational one without using ultrashort pulse duration. The nonresonant background signature of the sample is compared to those obtained with referenced materials such as fused silica and SF57 glasses. Such comparison provides then the quantitative evaluation of the mean $n_{2}$ value $\sim 5.72 \times 10^{-19} \mathrm{~m}^{2} \mathrm{~W}^{-1}$ for $\mathrm{TeO}_{2}-\alpha$ crystal. The obtained value is in excellent agreement with the results recently published by Duclère et al. ${ }^{13}$ by means of $\mathrm{z}$-scan technique in femtosecond regime, and with the THG data from Kim et al. ${ }^{22}$ collected on pure $\mathrm{TeO}_{2}$ glass. Additionally, we also accessed the in-plane modulation of $n_{2}$ of $\mathrm{TeO}_{2}-\alpha$ crystal. If M-CARS is commonly used to obtain the vibrational signature of samples, we demonstrated in this work that it can also be efficient to extract the real part of the nonresonant third order susceptibility. Such demonstration opens the way to spatial mapping of complex nonlinear samples including different molecular structures, as ceramics or glass-ceramics.

\section{ACKNOWLEDGMENTS}

We wish to acknowledge the support of French defense agency (Direction Générale de l'Armement) and the French national research agency (ANR) through the NEOSPRAM project (ANR14-ASTR-0014). This work benefited also of a financial support via the TRAFIC project (ANR-18-CE08-0016-01). Finally Z. Rajaofara wishes to thank the Région Nouvelle-Aquitaine for partial funding of his $\mathrm{PhD}$, within the frame of the NEMATUUM project.

\section{REFERENCES}

${ }^{1}$ P. D. Maker and R. W. Terhune, "Study of optical effects due to an induced polarisation third order in the electric field strength," Phys. Rev. 137, A801-A818 (1965).

${ }^{2}$ J. A. Armstrong, N. Bloembergen, J. Ducuing, and P. S. Pershan, "Interactions between light waves in a nonlinear dielectric," Phys. Rev. 127, 1918-1939 (1962).

${ }^{3}$ A. C. Eckbreth and T. J. Anderson, "Dual broadband CARS for simultaneous, multiple species measurements," Appl. Opt. 24, 2731 (1985).

${ }^{4}$ A. Zumbusch, G. R. Holtom, and X. S. Xie, "Three-dimensional vibrational imaging by coherent anti-Stokes Raman scattering," Phys. Rev. Lett. 82, 4142-4145 (1999).

${ }^{5} \mathrm{H}$. Kano and H. Hamaguchi, "Ultrabroadband multiplex coherent anti-Stokes Raman scattering microspectroscopy using a supercontinuum generated from a photonic crystal fiber," Appl. Phys. Lett. 86, 121113 (2005).
${ }^{6}$ M. Okuno, H. Kano, P. Leproux, V. Couderc, and H. Hamaguchi, "Ultrabroadband multiplex CARS microspectroscopy and imaging using a sub-nanosecond supercontinuum light source in the deep near infra-red," Opt. Lett. 33, 923-925 (2008).

${ }^{7}$ E. M. Vartiainen, K.-E. Peiponen, and T. Tsuboi, “Analysis of coherent Raman spectra," J. Opt. Soc. Am. B 7, 722-725 (1990).

${ }^{8}$ E. M. Vartiainen, H. A. Rinia, M. Müller, and M. Bonn, "Direct extraction of Raman line-shapes from congested CARS spectra," Opt. Express 14, 3622-3630 (2006).

${ }^{9}$ C. H. Camp, Y. J. Lee, and M. T. Cicerone, "Quantitative, comparable coherent anti-Stokes Raman scattering (CARS) spectroscopy: Correcting errors in phase retrieval," J. Raman Spectrosc. 47, 408-415 (2016).

${ }^{10}$ E. Capitaine, N. O. Moussa, C. Louot, C. Lefort, D. Pagnoux, J.-R. Duclère, J. F. Kaneyasu, H. Kano, L. Duponchel, V. Couderc, and P. Leproux, "Coherent anti-Stokes Raman scattering under electric field stimulation," Physical Review B: Condensed Matter and Materials Physics 94, 245136 (2016).

${ }^{11}$ E. Capitaine, N. O. Moussa, C. Louot, S. M. Bardet, H. Kano, L. Duponchel, P. Lévêque, V. Couderc, and P. Leproux, "Fast epi-detected broadband multiplex CARS and SHG imaging of mouse skull cells," Biomed. Opt. Express 9, 245-253 (2017).

${ }^{12}$ S. Yang and F. Ganikhanov, "Dispersion of the resonant nonlinear optical susceptibility obtained with femtosecond time-domain coherent anti-Stokes Raman scattering," Opt. Lett. 38, 4754-4757 (2013).

${ }^{13}$ J.-R. Duclère, T. Hayakawa, E. Roginskii, M. B. Smirnov, A. Mirgorodsky, V. Couderc, O. Masson, M. Colas, O. Noguera, V. Rodriguez, and P. Thomas, "Third order nonlinear optical properties of a paratellurite single crystal," J. Appl. Phys. 123, 183105 (2018).

${ }^{14} \mathrm{P}$. A. Thomas, "The crystal structure and absolute optical chirality of paratellurite, alpha- $\mathrm{TeO}_{2}$," J. Phys. C: Solid State Phys 21, 4611-4627 (1988).

${ }^{15}$ V. Rodriguez, M. Couzi, F. Adamietz, M. Dussauze, G. Guery, T. Cardinal, P. Veber, K. Richardsonc, and P. Thomas, "Hyper-Raman and Raman scattering in paratellurite $\mathrm{TeO}_{2}$," J. Raman Spectrosc. 44, 739-745 (2013).

${ }^{16}$ R. W. Boyd, Nonlinear Optics (Academic Press, 2008).

${ }^{17} \mathrm{D}$. Milam, "Review and assessment of measured values of the nonlinear refractive-index coefficient of fused silica," App. Opt. 37, 546-550 (1998).

${ }^{18}$ S. R. Friberg and P. W. Smith, "Nonlinear optical glasses for ultrafast optical switches," IEEE Journal of Quantum Electronics 23, 2089-2094 (1987).

${ }^{19}$ J. E. Aber, M. C. Newstein, and B. A. Garetz, "Femtosecond optical Kerr effect measurements in silicate glasses,” J. Opt. Soc. Am. B 17, 120-127 (2000).

${ }^{20}$ D. Lorenc, M. Aranyosiova, R. Buczynski, R. Stepien, I. Bugar, A. Vincze, and D. Velic, "Nonlinear refractive index of multicomponent glasses designed for fabrication of photonic crystal fibers," Appl. Phys. B 93, 531-538 (2008).

${ }^{21}$ J. Cimek, N. Liaros, S. Couris, R. Sepien, M. Klimczak, and R. Buczynski, "Experimental investigation of the nonlinear refractive index of various soft glasses dedicated for development of nonlinear photonic crystal fibers," Opt. Mater. Express 7, 3471-3483 (2017).

${ }^{22}$ S.-H. Kim, T. Yoko, and S. Sakka, "Linear and nonlinear optical properties of $\mathrm{TeO}_{2}$ glass," J. Am. Ceram. Soc. 76, 2486-2490 (1993).

${ }^{23}$ A. I. Kolesnikov, I. A. Kaplunov, S. E. Ilyashenko, V. Y. Molchanov, R. M. Grechishkin, M. A. Arkhipova, and S. A. Tret'yakov, "Optical parameters of paratellurite crystals," Physical Properties of Crystal 57, 909-911 (2012). 